\title{
The Asymmetric Priming Hypothesis
}

Welcome back to Ten Lectures on Diachronic Construction Grammar. The title of this lecture is "The asymmetric priming hypothesis". In the past four lectures, I have presented several corpus linguistic studies of constructions and how they changed over time. I have explained how I see the relation of grammaticalization theory and Diachronic Construction Grammar. I have discussed corpus linguistic methods that can be applied for the study of these processes. This lecture will be something of a change of pace. I won't be talking about diachronic corpus data in this lecture and I will present instead an analysis of synchronic corpus data and experimental behavioral data. You might ask yourself what psycholinguistic experiments can contribute to the study of language change.

Let me give you a preliminary answer to that question. The answer to that has to do with one of the central questions of usage-based linguistics, namely, how cognitive processes that operate in the here and now affect language in such a way that it changes in regular ways over time. In the very first lecture I have discussed ten basic ideas of Construction Grammar. The tenth one was the idea that language draws on domain-general socio-cognitive processes that include categorization, association, routinization, generalization, schematization, joint attention, statistical learning, analogy, metaphor and several others. We as human beings are equipped with a set of social cognitive skills that conspire to let us learn and use language.

Michael Tomasello expresses this idea in a way that is really simple and to the point (2005: 193): "Children acquire all linguistic symbols of whatever type with one set of general cognitive processes". As someone who is interested in language change, I want to find out how these processes operate in actual speech situations that influence how language changes over the long term.

In this talk, I want to take a closer look at one such cognitive process, namely a specific subtype of priming that has been called "asymmetric priming", which has been suggested as a force that shapes language change.

All original audio-recordings and other supplementary material, such as any hand-outs and powerpoint presentations for the lecture series, have been made available online and are referenced via unique Dor numbers on the website www.figshare.com. They may be accessed via this QR code and the following dynamic link: https://doi.org/10.6o84/mg.figshare.13691224. 


\section{asymmetric priming}
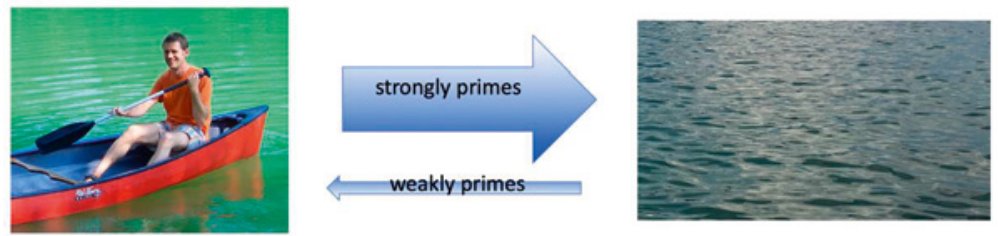

FIGURE 1

To get us started, take a piece of paper, take a pen and write down three words that come to mind when you hear the word paddle. One very frequent response that people write down is the word water. In other words, the word paddle primes you for the idea of water. It evokes the idea of water or at least it makes it easier to process the idea of water. By contrast, what do you imagine people will write down as their first associations when I give them the word water? Starting with water, you will think of drink, rain, sea, river and maybe flower, because flowers need water. But one item that you surely won't find among the first three words is the word paddle.

Paddle strongly primes water, but water only weakly, if at all, evokes the idea of a paddle. Now you know what asymmetric priming is, but you are perhaps wondering what it has to do with language change.

I will get to that in just a minute. First, let me give you an overview of this lecture. In the first step, I will introduce what's called the asymmetric priming hypothesis. I will outline what this hypothesis predicts for behavioral data and for corpus data. Then I will present an experimental study that addresses these predictions. After that, I will continue with the second study that tests the asymmetric priming hypothesis on the basis of corpus data. In the fourth part, I will offer some tentative conclusions. Without further ado, what is the asymmetric priming hypothesis?

The hypothesis has been proposed in a programmatic paper written by Jäger and Rosenbach (2008). In this paper they state the following:

We argue that the psycholinguistic mechanisms of PRIMING may account for the empirical observation that grammaticalization processes typically proceed in one direction only. 
As I have discussed in previous lectures, grammaticalization is concerned with the emergence of grammatical forms, and it describes a number of different interlocking types of change that all proceed in one direction only. Full forms with lots of phonetic substance develop into forms with less phonetic substance. Forms that are only loosely integrated in discourse develop into forms that are syntacticized, into forms that are further compressed into morphological constructions. Concrete meanings are becoming more abstract and schematic, but not the other way around. The changes in grammaticalization are asymmetric. There is one direction and we see the changes moving in that direction only. Jäger and Rosenbach argue that they have an explanation for unidirectionality. They claim that there is a cognitive process, something that happens in conversation and acts synchronically on the minds of speakers, that could explain why in the long run changes happen in languages the way they do.

Jäger and Rosenbach elaborate on this first statement:

Very generally, the prediction is that in any reported case of change, where the development goes unidirectionally from A to B, A should prime B but not vice versa.

When we have a grammaticalization process in which A turns into B, then there should be an asymmetric priming relation, so that A primes B, but not the other way around. That is a claim that can be tested empirically in an experiment. Let me show you a concrete example of what Jäger and Rosenbach are proposing. Let's look at a case where we have A changing into B, and let us consider the predicted priming relation between the two.

Imagine that you are out on the road. That stick figure here on the slide, that is you, and you meet a friend of yours who tells you "I am going to the station". The asymmetric priming hypothesis would predict that even though your friend just talked about the act of walking, you would be likely to think about your friend's future and his actions in the future. What is your friend going to do? Is he going to catch his train? Where will he be going and what will he be doing once at his destination? The utterance "I am going to the station" triggers all these temporal associations. Conversely, if you're talking on the phone and the person you're talking to tells you "Oh, I gotta go. I am going to miss my train", your friend's utterance encodes a future event. According to the asymmetric priming hypothesis, talking about time should not make you think about physical motion, at least not as much. You're talking about a future event and you can think about that in its own right, without thinking about motion. This, in a nutshell, translates into the following research question: Does go prime be 
I'm going to the station.

Lexical going primes grammatical be going to.
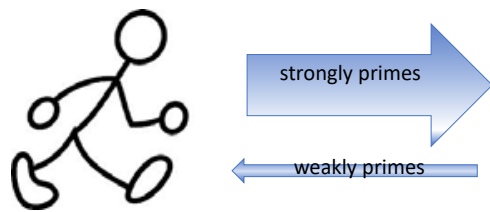

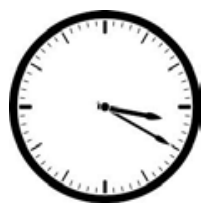

Grammatical be going to does not prime lexical going.

I'm going to miss my train.

FIGURE 2

going to, but not vice versa? According to what I have just told you, we have lexical going, which should prime grammatical be going to, but grammatical be going to should not prime lexical going. Now, we are talking about actual linguistic forms that we can integrate into experimental stimuli that we can present to subjects. Doing so allows us to check whether the predictions of the asymmetric priming hypothesis actually hold up.

Does go prime be going to, but not vice versa? If it does, asymmetric priming might be an explanation for one aspect of unidirectionality in grammaticalization, namely, unidirectional semantic change. That is just one aspect of unidirectionality, but it is an important aspect. It would be one big step forward if we could explain this unidirectional aspect of grammaticalization in terms of a psychological process that operates in the here and now.

In order to find out whether this is actually the case, David Correia Saavedra and I designed an experiment with which we wanted to assess whether or not the asymmetric priming hypothesis makes the right predictions about language processing in real-life speakers. What did we do?

We constructed a database of 20 elements that in English have both lexical uses and grammatical uses. Basically any grammaticalized form that you can find has a lexical counterpart of some sort. There are exceptions like demonstratives for instance, which often do not have lexical counterparts. But for most of grammaticalized forms, there are correspondences such as the ones that you have on this slide here. The English verb have has grammatical uses as in "I have solved the problem", and it has lexical uses such as "I have a problem". Lexical have encodes possession, and grammatical have encodes the perfect. 
Grammatical have has other functions as well, as in "I have someone service the car", where it has causative meaning. Another pair in our database consists of lexical keep and grammatical keep. Lexical keep means "Keep your jacket on", maintain it in a certain position. "Keep walking" means carry on walking. It aspectually modifies the meaning of the lexical verb.

Besides verbs, our database contains other parts of speech, including forms that start out as lexical adjectives and then become a part of a grammatical element. The adjective long is an adjective, and you can use it lexically in expressions such as "as long as a python". It also appears as part of the clause connector as long as: "As long as you do not get caught, you can do anything". We can reconstruct the semantic development that went from extension in space, i.e. how long something is, to a temporal extension, as in "That was a long story" to conditional meaning, as in "as long as you do not get caught". The grammaticalization path of long is thus a semantic development from spatial meaning to temporal and conditional meaning.

We created a database with 20 such pairs, and we designed a test of the asymmetric priming hypothesis in order to find out whether there are any asymmetries in the ways lexical elements prime their grammaticalized counterparts and vice versa. I mentioned earlier the example of going and be going to, saying that going should prime be going to, but not vice versa. The same phenomenon should be observable across all pairs in our database, so that have a problem should prime have solved the problem, but not vice versa. A long python should prime as long as, but as long as should not prime a long python.

We used an experimental method that is known as the maze task. Since this procedure is not so common, I brought you a little illustration. In the experiment, participants have two response buttons, one on the left and one on the right. They are given the following instructions. They are told that they will see two words displayed on the screen, and that their task is to select one of the words that they see. The words that they select should combine to form a meaningful sentence. The next slide shows an example, so you can actually experience directly what the participants saw.

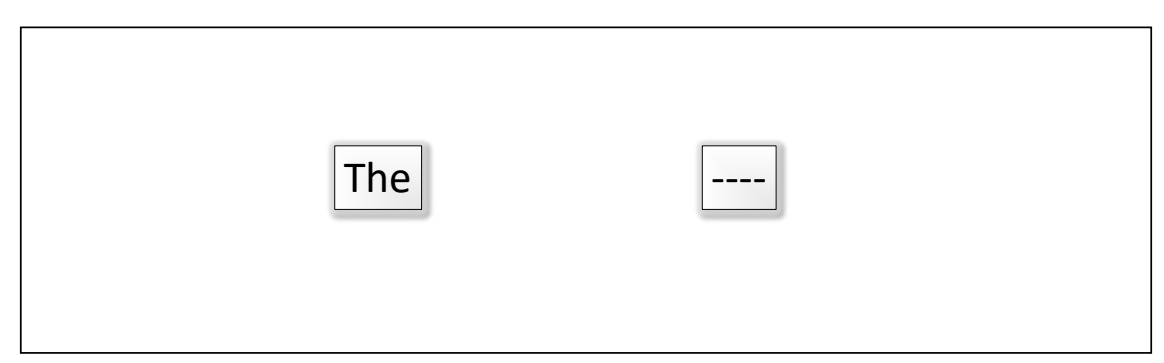

FIGURE 3 
Here's the first screen. There are two fields and because this is the first pair, there is only one word and the other box shows only three hyphens. Here our participants have to press the button for the on the left.

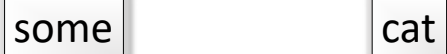

FIGURE 4

The next screen shows the word some and the word cat. After the, what word do you have to choose to continue a grammatically functioning sentence? You have to choose cat, so the cat.

not

is

FIGURE 5

Here we have not and is. Which one do you like better? Is? Ok.

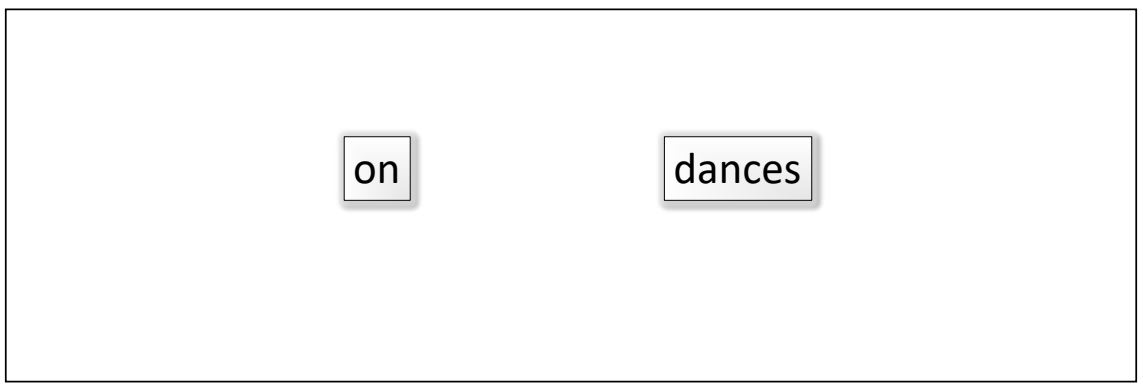

FIGURE 6 
We have on and dances, so The cat is on.

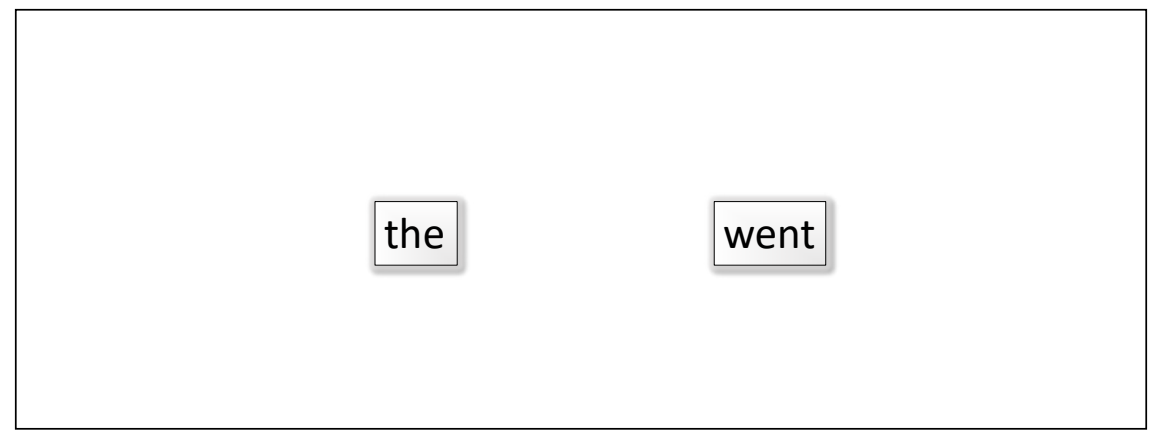

FIGURE 7

The. Ok.

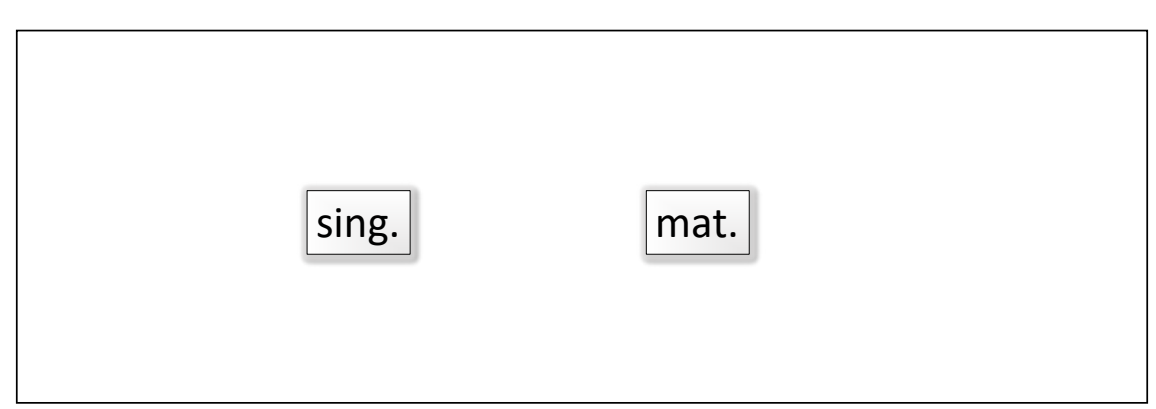

FIGURE 8

Mat. This gives us one of philosophy's most famous sentences, The cat is on the mat. What you have just done was exactly what our participants did, except they had to do it for a much longer time, and of course with other sentences. The maze task is a mixture between a self-paced reading design, where you read and you click as soon as you have read something, and a forced-choice task, i.e. there are two options, and you have to pick one.

Subjects see screens that present two options, but only one of them makes sense, given the prior context. Every time they press a button, we measure reaction times, so we know exactly how long it takes them to pick the right word. This can be exploited for the analysis of asymmetric priming effects.

For our experiment we recruited 200 speakers of American English via an online platform. These people could sign up online and do this task in their own homes. That comes with advantages and disadvantages. The disadvantages are that we do not know who they are, we do not know if they were checking their social media while they were doing the experiment, and we do 
four conditions

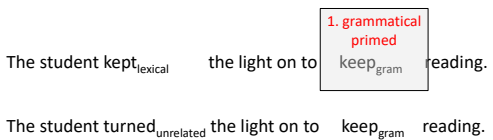

The student kept $\mathrm{gram}_{\text {checking facebook to }}$ keep lexical $_{\text {a }}$ up to date.

The student was unrelated checking facebook to keep lexical up to date.

FIGURE 9

not know how much attention they were paying. However, the advantage is that you can recruit many participants in a very short time.

The 200 speakers of American English clicked their way through 40 stimuli sentences, 20 of which contained a primed word. Those were the critical stimuli, the rest were fillers. Only fully correct responses entered the analysis. If someone made a mistake and selected the wrong word, we threw away the data point. We only took sentences in which the participants got every single word right. The overall experiment took them about 12 minutes. We measured reaction times, i.e. how fast they selected the correct element across four different conditions, that is four different versions of the stimuli. This is something that I need to explain in detail.

In the first condition, we collected responses to a grammatical element that had been primed by its lexical counterparts. Let me give you an example for that kind of sentence. If we have a sentence like "The student kept the light on to keep reading", we have two instances of the word keep in the sentence. The first is lexical, "the student kept the light on" and then there is "to keep reading". We have the grammatical version of keep in the second half of the sentence. This would be what we call the grammatical primed condition, that is, we are measuring how quickly participants respond to grammatical keep at the end of the sentence, and that grammatical keep has been primed by lexical keep. According to Jäger and Rosenbach (2008), this should be easy because there is a positive priming effect from the lexical source word, which should give people an advantage.

The second condition is similar, except here the grammatical word is not preceded by its lexical source, but rather by an unrelated word. The sentence would simply be "The student turned the light on to keep reading". In this context, grammatical keep does not have the advantage of being primed by its lexical source. 


\section{four conditions}

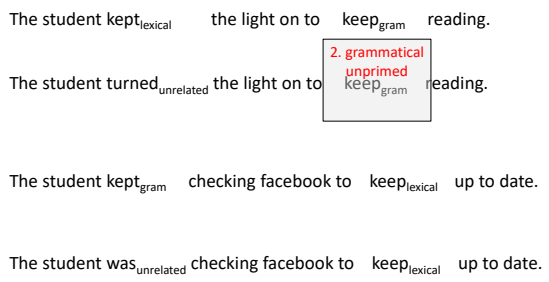

FIGURE 10

\section{four conditions}

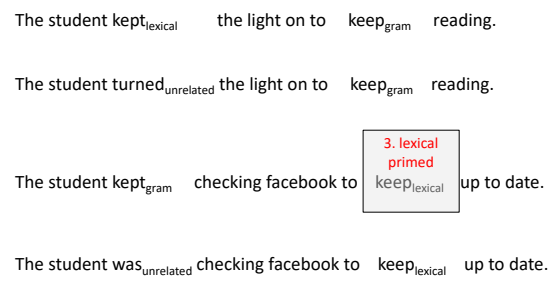

FIGURE 11

\section{four conditions}

The student kept lexical the light on to keep gram reading.
The student turned
unrelated

The student kept $t_{\text {gram }}$ checking facebook to keep lexical up to date.

The student was unrelated checking facebook to $\begin{gathered}4 . \text { lexical } \\ \text { unprimed } \\ \text { keep }_{\text {lexical }}\end{gathered}$ up to date.

FIGURE 12 
asymmetric priming effects?

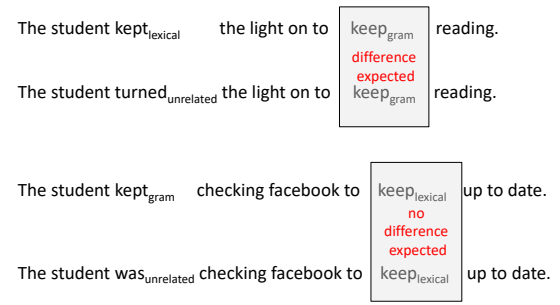

FIGURE 13

In conditions three and four, we measured responses to lexical variants. In "The student kept checking facebook to keep up to date", we have the grammatical variant of keep first, the student kept checking, and keep up to date in the second half of the sentence. We thus measured responses to lexical keep that had been primed by its grammatical counterpart. According to Jäger and Rosenbach (2008), that type of priming should not yield an advantage. There is no priming predicted from grammatical keep to lexical keep.

The last condition is exemplified by sentences like "The student was checking facebook to keep up to date". This is the lexical unprimed condition, in which lexical keep is not primed by a previous element in any way.

What priming effects are we predicting? On the asymetric priming hypothesis, keep in the first sentence should have a processing advantage over keep in the second one. In the second pair of conditions, a different prediction holds, namely, since grammatical keep is not expected to prime lexical keep, condition three and four should yield the exact same results.

For the analysis, we used a regression analysis design. Our dependent variable in this case was a continuous variable, namely, the reaction time. How quickly did people press the right button? The analysis includes several predictor variables. The first of course is the presence of priming. We also controlled for the fact whether a stimulus was grammatical or lexical. Since we are corpus linguists, we also included the text frequency of the items as a variable, because it is known that higher frequency items are more easily processed. We further included several control variables, namely gender, age and handedness of our participants.

We used a mixed-effects regression design and included random intercepts for the participants. Some people are just quicker than others. Some people just 
had their coffee. Others had a rough night. We control for that with that factor. We also included a random factor for the test item, which allows the same kind of variation for all the different elements in the database. We included an interaction effect between the priming variable and the variable that distinguishes between lexical and grammatical. This is of course the heart of the asymmetric priming hypothesis: Does priming work differently, depending on whether we are measuring the response time for a grammatical element that has been primed by its lexical counterpart, or whether we are measuring the response time for a lexical element that has been primed by its grammatical counterpart? That is the prediction that the asymmetric priming hypothesis makes.

We also tested for an interaction effect between priming and frequency, because frequent items may not need priming to be processed very quickly. When I give you a very rare word, for instance, procrastinate, the next time you hear procrastinate, this memory of hearing this word just recently pops into your mind and you can process it rather quickly. Contrast that with when I pronounce the word the, which you hear so often. Hearing it one more time won't change your response to it the next time you hear it. That is captured by an interaction term between priming and frequency. High frequency elements do not profit from priming as much as low frequency items.

What came out of the analysis? We computed a full regression model that includes all the variables that I have mentioned. None of the control variables that we included, i.e. age and gender and handedness, change the results in any way. The analysis further does not show evidence for an interaction between priming and frequency, so we remove that from the model as well.

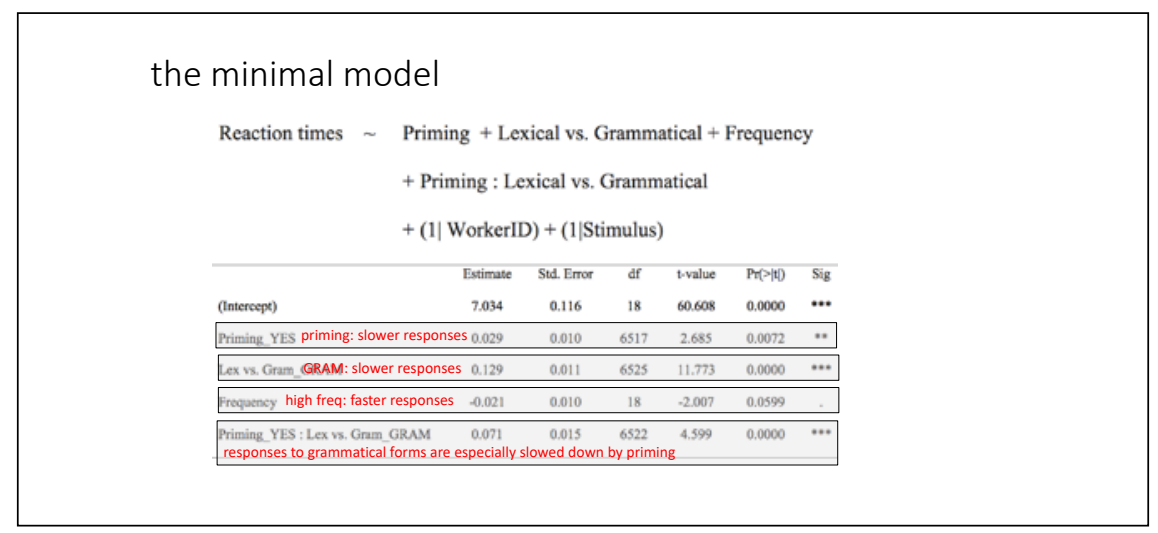

FIGURE 14 
The exclusion of those variables results in a minimal model that only retains the variables that yield a significant effect. Those variables are the priming variable, the lexical versus grammatical variable, text frequency, and the interaction of priming, and lexical and grammatical. Let me walk you through the effects that we see in the table on this slide.

The first effect that I want to talk about concerns the priming variable. It turns out that forms that have been primed by their lexical or grammatical counterpart are not verified faster, but slower. That is the opposite of what one might predict. Primed forms have generally slower response times.

Another effect concerns the lexical versus grammatical variable. It turns out that grammatical forms in general have slower responses. That is equally surprising, because grammatical forms tend to be highly frequent. It should be very easy for the human processor to recognize these forms. This means that we have two rather unexpected results.

The next effect concerns frequency. High frequency items yield moderately faster responses. It is an established finding that high frequency forms are processed more easily. We find that in our data as well.

Then is the asymmetric priming effect. Priming interacts with the lexical versus grammatical variable in such a way that responses to grammatical forms are especially slowed down when those grammatical forms are primed. In other words, in "The student kept the light on to keep reading", that grammatical form of keep at the end of the sentence is the worst condition in terms of how quickly participants can process it.

How does that line up with our expectations? As you remember, we expected no difference between lexical primed and lexical unprimed. That prediction turned out to be correct, but as far as the predicted difference between grammatical primed and grammatical unprimed is concerned, we expected an advantage of the grammatical primed condition. Not only did we not find that, we found that responses in the grammatical primed condition are slower than responses in the grammatical unprimed condition. The difference is statistically highly significant.

On this slide you see a visual representation of the response times. We expected no difference between lexical primed and lexical unprimed. Indeed, if you look at the box plots there side by side, you can see that they are very similar. The average is around goo milliseconds, and there is no recognizable difference. There is, however, a difference between the primed and unprimed grammatical conditions. The difference is significant, but it is in the direction that was not predicted by the asymmetric priming hypothesis.

So, does go prime be going to, but not vice versa? We observed asymmetric priming effects, but they do not work in the way we expected. Priming between 


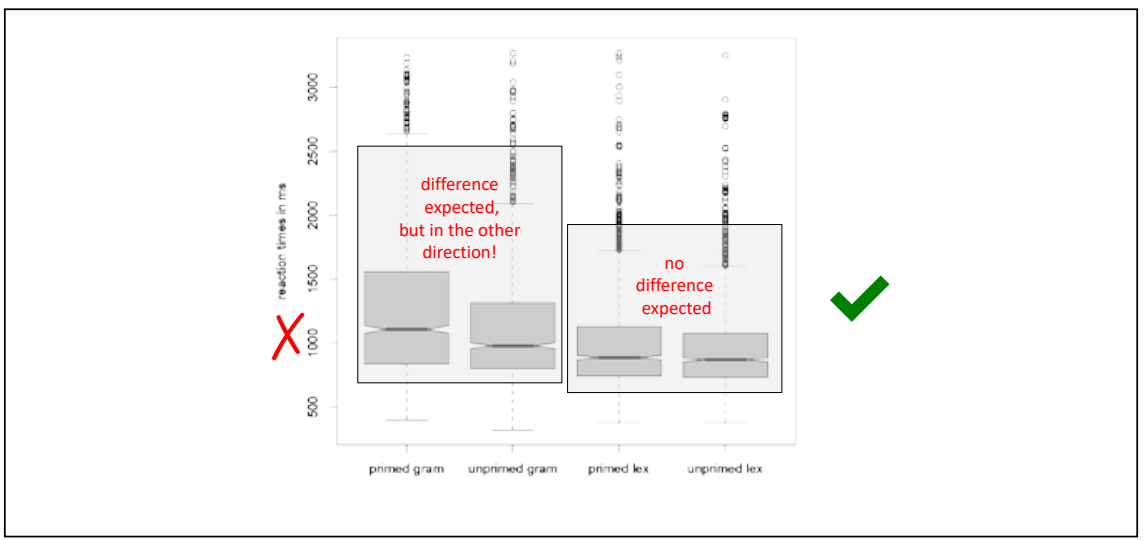

FIGURE 15

lexical forms and their grammaticalized counterparts is negative. It takes longer to process a given form when it has been primed. This negative priming particularly affects the processing of grammatical elements. To put it into a formula, go slows down be going to more than vice versa. Experimental evidence thus seems to contradict the asymmetric hypothesis.

Before giving up on it, we wanted to consider another methodological perspective and for that I turn back to corpus-based evidence. When we examine the way people actually use language, the way they write and the way they talk, are grammatical forms in corpus data preceded by the lexical sources more often than one might expect? Do people construct sentences like the one that we did? Or do they just not do that? We investigated these questions with the help of methods from distributional semantics.

At this point I am afraid I need to take a step back and say a few words about distributional semantics in general. I will eventually come back to asymmetric priming, but there are first some issues that I need to get out of the way. Please bear with me.

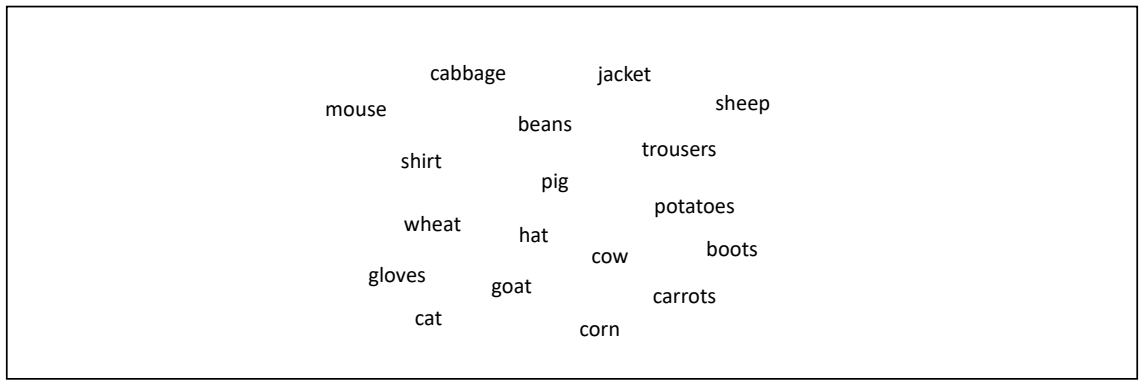

FIGURE 16 


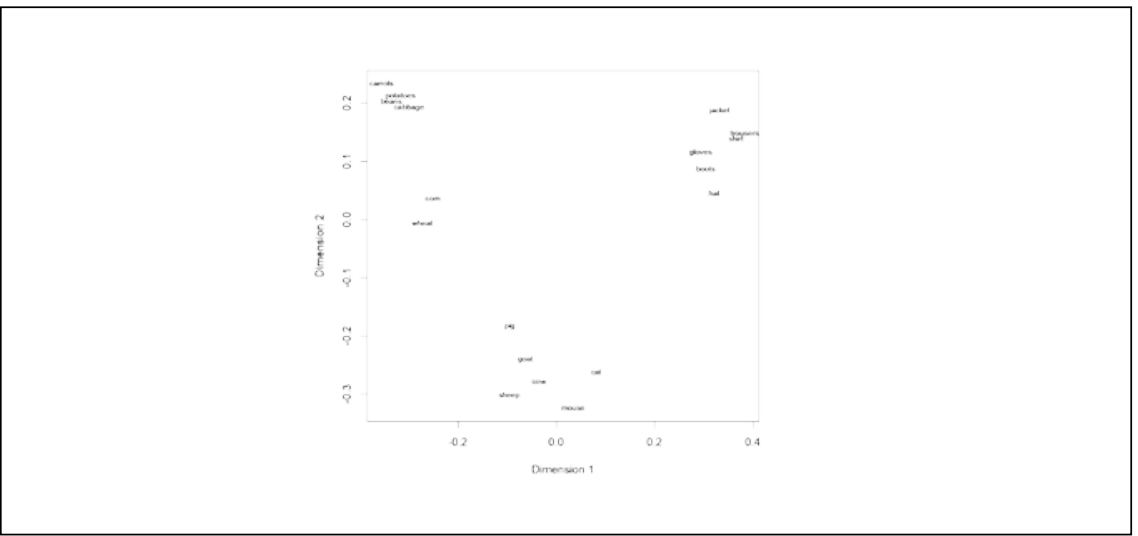

FIGURE 17

On this slide, you see different words that describe things that could be found on a farm. We have cabbage, beans, pig, cow, potatoes. We have wheat. We have clothing items like a hat, gloves or boots, things that a farmer might wear. These words are semantically related to each other, but of course they are not all equally similar to each other. In fact, if you were to give these words to a human observer, they would probably tell you that the words fall into three different categories, namely animals, vegetables and clothing items. The words carrots and potatoes instantiate vegetables. The words gloves, hat and shirt are clothing items. We have animals such as pig, cow and sheep. Distributional semantics is a computational way of studying semantic similarity across different words.

On this slide here, you see a visualization that looks a lot like the grouping of words that I showed on the previous one, except this one has not been made by a human being. Here we have the result of a computational analysis, in which a computer has categorized our farm words in a way that is quite similar to what a human being would have done. We have the vegetables in one corner, the upper left, we have the clothing items to the right, and we have the animals further down in the middle.

One thing you see is that in some way the computer here has been a little more insightful even than my own intuitive analysis, because you see that the computer thinks that corn and wheat aren't really prototypical vegetables, so corn and wheat form their own grain kind of category that is a little apart from the other vegetables. This is evidence that this method actually works.

But how does this work? How does the computer know that some words are related and others aren't? And what work steps are involved? I would like to walk you through the steps that are involved, so that you have a sense of what lies behind this. 
The first work step that is involved is that we choose a vocabulary of key words that we are interested in. We retrieve concordances of these words from a corpus, which gives us access to frequencies of items in their context. Let me show this in practice.

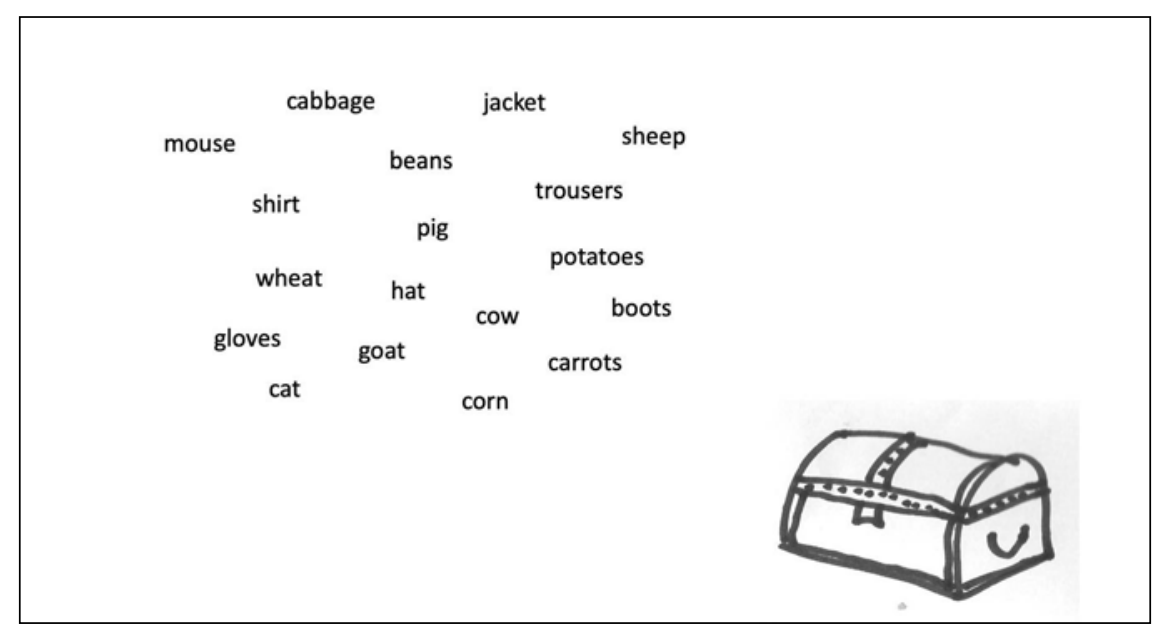

FIGURE 18

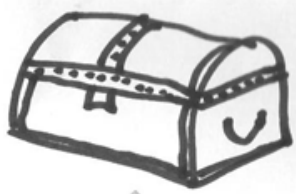

much hope there. He has his established. Within the mountain is to get a dead mountain driving a donkey laden with he wouldn't be a tethered was in good spirits. The her a tart or because her pet usually from a combination of And have that lecherous old goat

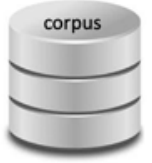

clinic on Fridays. I hope you community, this leads to continual down from a mountain -- it simply fodder. As he passed our party from choice. He went into had been sacrificed at the shrine had gone missing ; she always hair , cotton and jute, and hanging round my door 


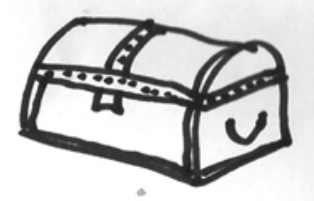

much hope there. He has his established. Within the mountain is to get a dead mountain driving a donkey laden with he wouldn't be a tethered was in good spirits. The her a tart or because her pet usually from a combination of And have that lecherous old

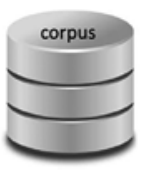

clinic on Fridays. I hope you community, this leads to continual down from a mountain -- it simply fodder. As he passed our party from choice. He went into had been sacrificed at the shrine had gone missing ; she always hair, cotton and jute, and hanging round my door

FIGURE 20

In the example I have shown, the vocabulary that we are using as the basis for our study is this set of farm words. In my native language German, there is a word for vocabulary that translates into treasure of words, "Wortschatz", which why you see the treasure chest here.

All elements should go into the treasure chest. Once we have the vocabulary, we can choose a corpus and retrieve concordances for all of our vocabulary items. In this case, we retrieve several concordance lines for the word goat. You see the keyword goat in the middle and a couple of words to the left and right. This data undergoes several processing steps. In the first processing step, grammatical words and other high frequency items are removed from the concordances. Those are the words that I have shown in grey on this slide. Pronouns like he or deictic elements like there or articles like the, all of those are deleted so that what remains are really just the contentful lexical items like Friday, community or fodder that form the context of goat to the left and right, and that presumably tell us something about the key word.

These are the words that the word goat collocates with.

All of these context items are collected in what we can call "a bag of words". Why is it called a bag of words? That is a technical term from corpus linguistics. It is called a bag of words, because in a bag all elements are mixed up and there is no linear structure to them anymore. In a sentence or in authentic language use, words follow each other in a linear way, but once you put them in the bag of words, you really only know how frequent they are, not how they are usually ordered. 


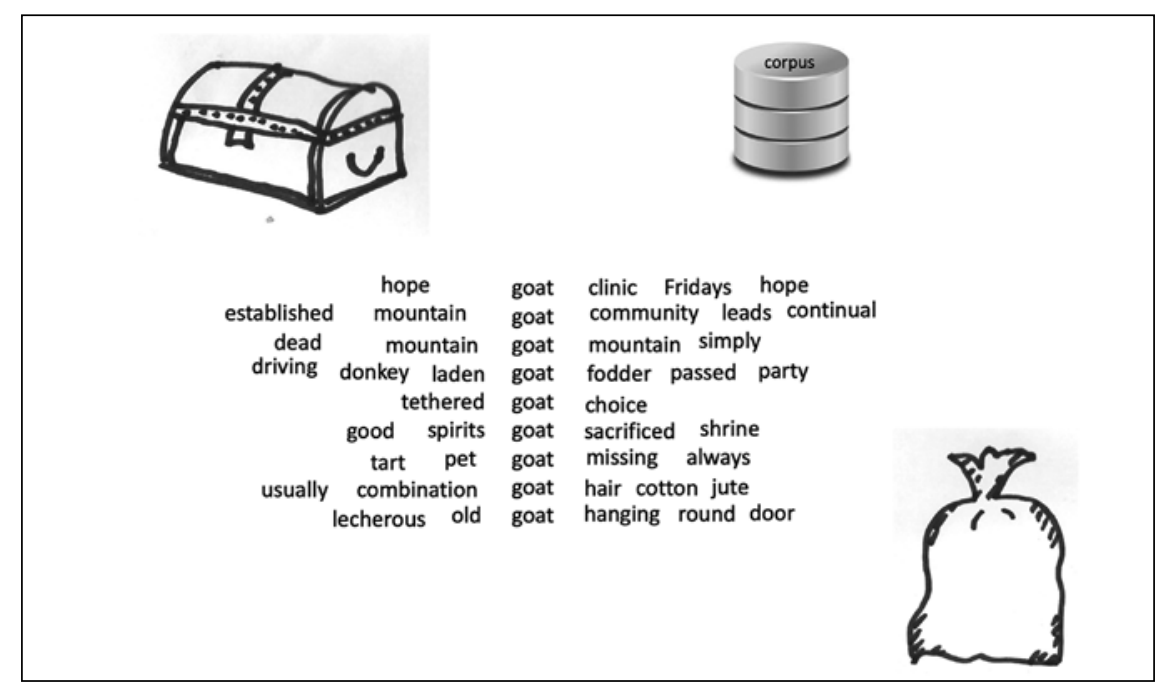

FIGURE 21
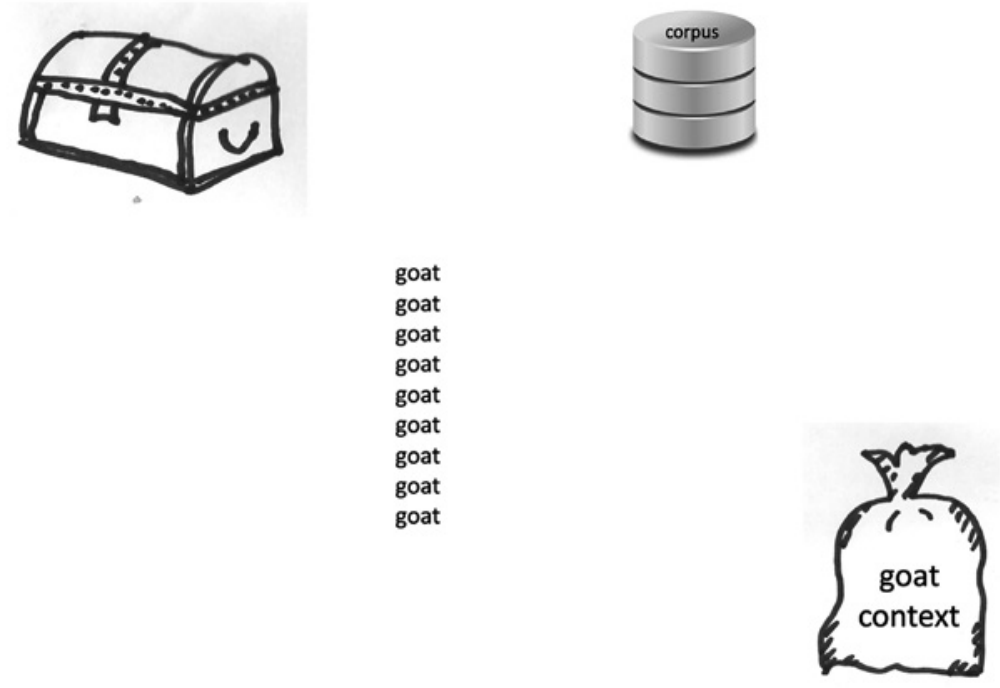

FIGURE 22

A bag of words like this can be easily transformed into a frequency word list. In the list on this slide, you see the most frequent elements that occur with the word goat. In the corpus that I used, the word mountain appears as the most frequent collocate. It is no coincidence that the word goat appears as a context item of itself. There is milk. There is cheese. The word sheep is a frequent 


\begin{tabular}{|c|c|c|c|c|c|c|c|}
\hline CONTEXT ITEM & FREQUENCY & CONTEXT ITEM & FREQUENCY & CONTEXT ITEM & FREQUENCY & CONTEXT ITEM & FREQUENCY \\
\hline mountain & 48 & milk & 119 & pig & 84 & shirt & 150 \\
\hline goat & 32 & cow & 80 & wild & 27 & pair & 133 \\
\hline milk & 30 & mad & 39 & head & 24 & jacket & 123 \\
\hline cheese & 20 & stupid & 38 & pigs & 23 & white & 108 \\
\hline sheep & 13 & disease & 34 & iron & 20 & black & 107 \\
\hline meat & 9 & silly & 28 & says & 17 & trousers & 80 \\
\hline horns & 8 & parsley & 26 & farm & 16 & wearing & 67 \\
\hline antibodies & 8 & sheep & 21 & meat & 14 & shoes & 60 \\
\hline black & 8 & calf & 18 & farmer & 14 & grey & 58 \\
\hline gets & 7 & per & 17 & food & 13 & blue & 55 \\
\hline hens & 7 & sacred & 17 & fact & 13 & wore & 54 \\
\hline eat & 6 & say & 16 & dog & 13 & boots & 51 \\
\hline tiger & 6 & little & 16 & thought & 12 & dressed & 48 \\
\hline head & 6 & dairy & 15 & prices & 12 & wear & 48 \\
\hline hand & 6 & bull & 14 & pot & 12 & cotton & 44 \\
\hline
\end{tabular}

FIGURE 23

collocate. There are meat, horns and other items that we associate with goats. These frequency lists are the basic information the computer works with. They are representations, if you like, of the semantic profile of a word. We produce these frequency lists not only for one word that we are interested in, but rather for all the others that we have as well.

Here are three more frequency lists, one for cow, one for pig, and one for the word trousers. The reasoning goes that words with similar frequency lists should have some semantic relation between them. When we examine these frequency lists on the slide, we find, for example, that goat has the word milk as a frequent collocate. Also, cow has the word milk as a frequent collocate. Goat has sheep as a frequent collocate, and so does cow.

You get the basic point. Words with similar meanings should occur with similar collocates at similar frequencies. When we compare goat, cow and pig, we should see a relatively high degree of similarity, as opposed to contrasting goat with trousers. The most frequent collocates of trousers are shirt, pair, jacket, white, black, trousers, and wearing. No meat, no horns and no milk. It has a different semantic profile. Words with similar frequency lists have some semantic relation, which can be one of synonymy, antonomy, partonomy, and some other -onymies that would come into play there.

That is the first work step: We choose a vocabulary of key words and we retrieve frequencies of context items from a corpus. That is what I just described to you. 
The second work step is an arrangement of these frequency lists in a table of frequencies that lists all the vocabulary items and how often they co-occur with all the context items. This slide shows the vocabulary items in the columns, the context items will appear in the rows. Let me show you how this works. We have our vocabulary items like goat, cow, pig, trousers, jacket, boots and others.

Then we have all context items from our bag of words. We have the table of vocabulary items and the context items.

In the cells of the table, we have the co-occurrence frequencies of each vocabulary item with each context item, that is, in the table, we have, for instance, the information that the word goat co-occurs with the word cheese 20 times. Some cells in the table are o, because not all vocabulary items occur with all context items. The word pig in this sample does not occur with the word grey. Goat occurs once with grey. Cow also does not occur with grey. That tells you not only about what kind of words occur frequently with each other, but also where there is an absence of co-occurrence.

That information allows us to compare pairs of vocabulary items. We can use a mathematical measure to determine how similar or how different they are. We can carry out pairwise comparisons, subtract the values in the cells from each other and arrive at a measure of dissimilarity, a measure of distance of these items from each other. Earlier this morning I talked about how this can be done with complement-taking verbs that have different profiles with regard to the syntactic structures that they occur with. Here we perform the same analytical step, except that we are dealing with a table that is much larger. We

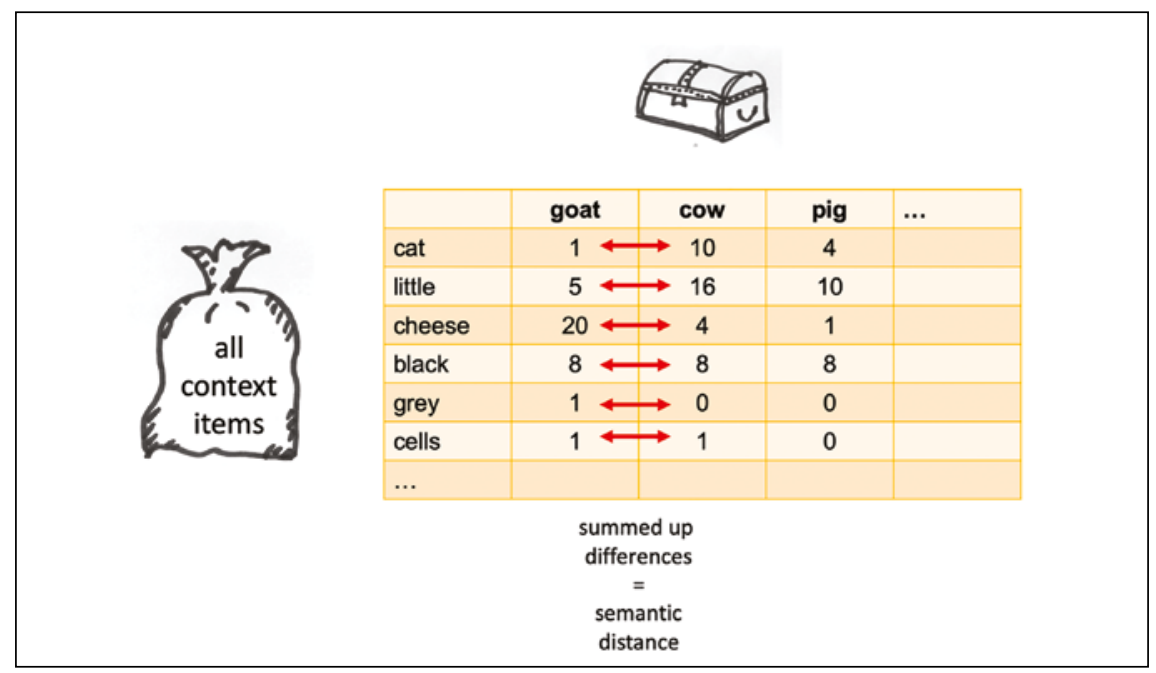

FIGURE 24 
do not only compare five different linguistic units, but rather thousands of different words that are the context items of goat and cow.

We can take the absolute differences, we can sum them up and that would give us a measurement of how semantically distant or similar two items are. We would expect a larger difference for the pairing of goat and trousers because one is an animal and the other is a clothing item. We would expect a smaller distance measure for goat and cow, because both are animals.

So far, our table contains raw co-occurrence frequencies of vocabulary items and context items. These frequencies cannot be taken as such. They need to be transformed with a collocation measure. Let me explain why.

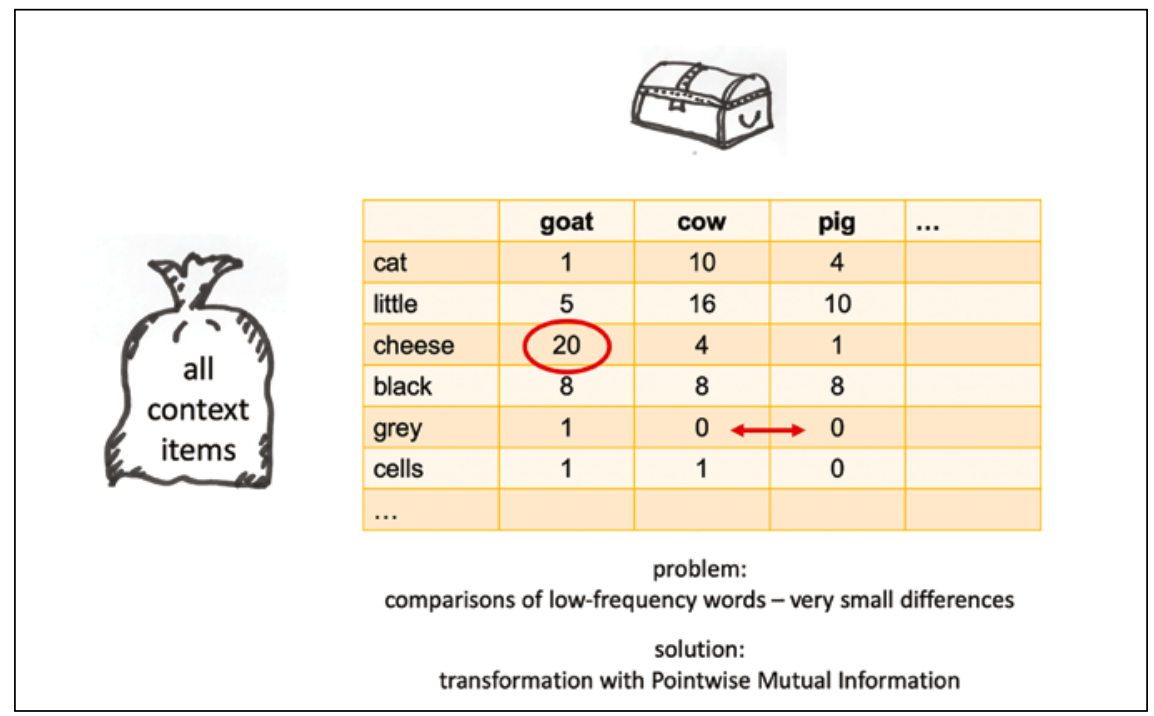

FIGURE 25

Comparisons between low frequency vocabulary items will involve many cells in this table that are o. Let's say that our vocabulary contains two very rare verbs: procrastinate and exempt. Both of them have for many context items the value $o$, which means that there are many cells where the two verbs are identical. This may yield the false impression that the two verbs are semantically very similar. However, procrastinate and exempt are not semantically similar. They're just similarly infrequent. That is why we need to perform a transformation of these raw frequencies in order to arrive at a more realistic assessment of how semantically similar or dissimilar two items are. This can be done with Pointwise Mutual Information, a measure of collocation which I would like to explain today in a little more detail.

Let me take a concrete example. In this table, we have 20 co-occurrence instances of goat and cheese. Is that more than what we would expect by 
chance? Is that about what we would expect by chance? Or is this actually less than what we would expect, given the basic frequencies of the word goat and the word cheese? Pointwise Mutual Information can give us an indication of that.

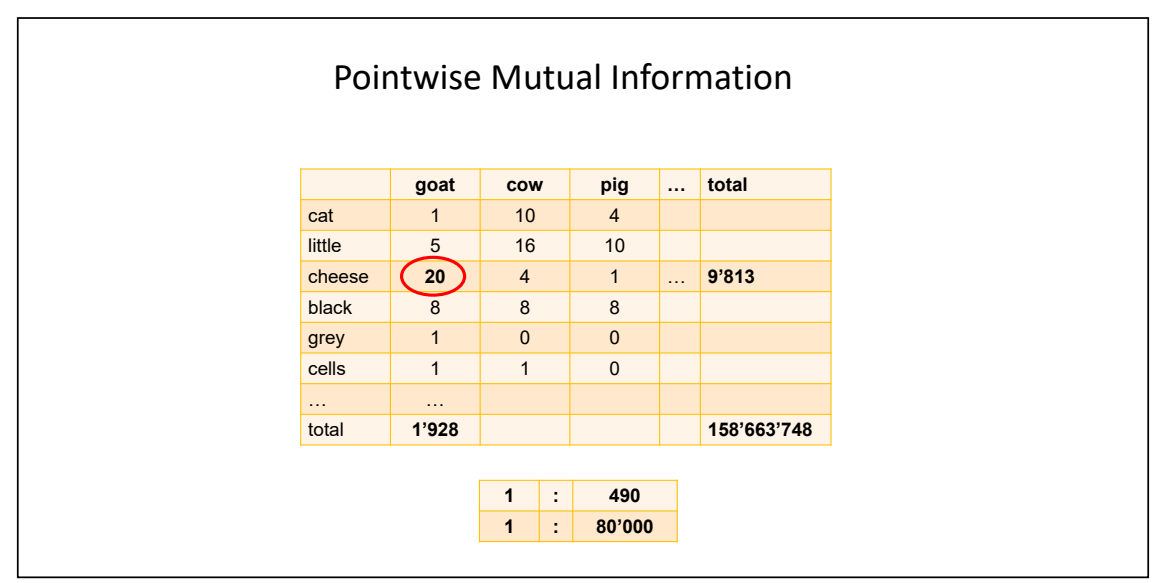

FIGURE 26

In order to compute a value of Pointwise Mutual Information, we need the marginal frequencies of this table here. We have all the instances of goat, which are about 200o. We have all the instances of cheese, which are about 9800. Then there is a very large number in the lower right corner of the table. That number indicates that we have about 16o million collocation pairs in the table. Point Mutual Information performs a comparison of ratios. Is 20 to 9800 about the same, or more or less than 1900 to 160 million? The ratio up 20 and 9800 is about 1:49o. The ratio of 1900 to 160 million is 1:80,00o. That means goat cheese is heavily over-represented in the data. This makes sense because we know that goat cheese is a lexical item that denotes a specific type of cheese.

The third work step is thus a transformation of the raw frequencies with Pointwise Mutual Information (PMI). This brings us to the fourth and final step, namely the visualization of the data. Once we have the table with its PMI values, we can transform that data into a visualization like the one I showed earlier with the farm words. That kind of data yields a display in which semantically close words are shown in very close proximity, and semantically distant words are further away from each other. Before we get back to asymmetric priming, there is one more issue that I need to explain. In order to answer our research question, we needed to turn to an extension of the general idea of distributional semantics.

In the graph you saw earlier, every item in the graph represented a word type, that is, there are several hundred concordance lines of the same word that 
determine in the end where on the graph a certain word appears. If you want to compare lexical going and grammatical be going to, those two constructions actually have the same form going. That means that this type-based approach is only of limited use. However, there is an extension of the technique: tokenbased semantic vector spaces. That approach can reveal semantic differences between word tokens of the same type. Let's say that we have a number of key word tokens, each of which is represented by one concordance line. Through the use of token-based semantic vector spaces, these concordance lines can be compared against each other, and we can investigate differences in meaning of the same key word.

How can we do that? If we base the analysis only on the words that are contained in the concordance lines, we have very little material to go on, which would be problematic. We have to find a way to make the concordance lines as informative as possible. The solution is to use not only the collocates of the key word as such, but in fact collocates of collocates. Let me explain how this works.

What we need for that are basically two data resources. The first resource is a type-based semantic vector space of the kind that I showed you just a minute ago, the one with the farm words, except this one holds a much larger vocabulary. It contains about 20,000 key words and 20,000 context items. We thus have a large vocabulary that we have characterized in terms of their collocates. That is our first resource.

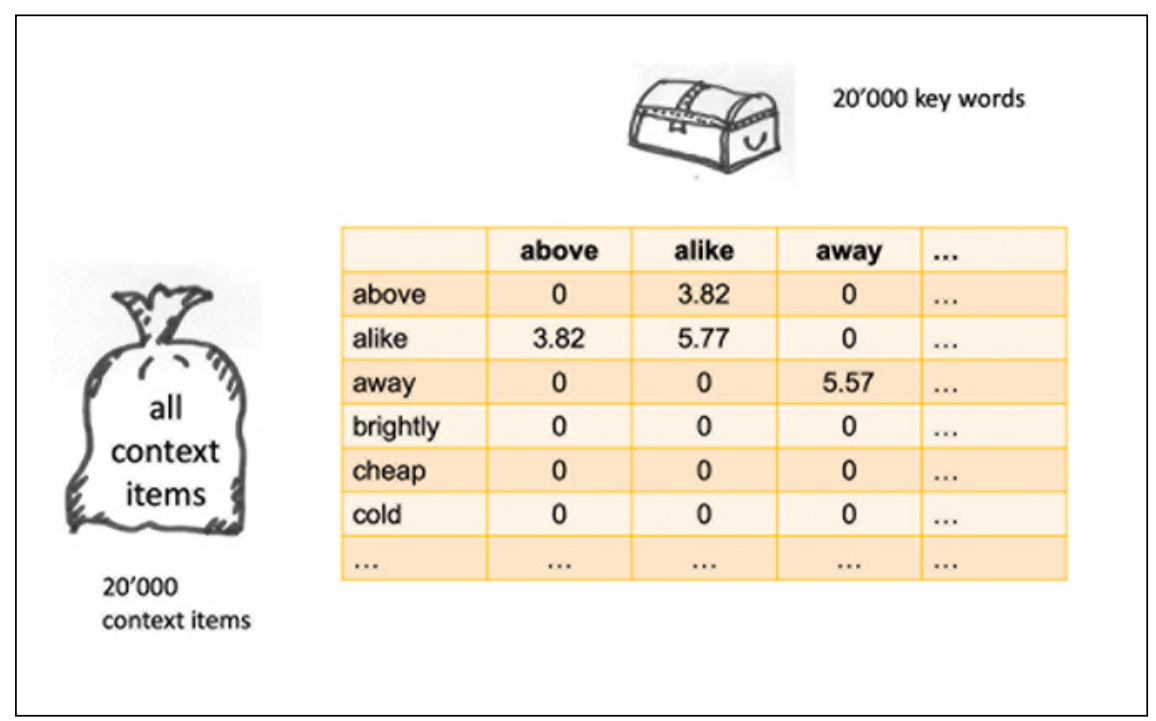

FIGURE 27 


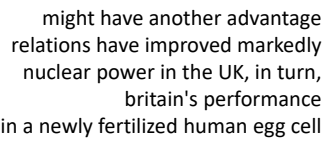

might have another advantage relations have improved markedly britain's performance in a newly fertilized human egg cell drugs are toxic materials

the change of government last weekend uranium reserves are finite

the war has been worse

DNA is the substance of which

FIGURE 28

The second resource consists of concordance lines for a word that we would like to model. Here we have a short concordance of the word since. Let me just read the first example, "might have another advantage since drugs are toxic materials", and the second "relations have improved markedly since the change of government last weekend". What we are trying to do is to create a semantic vector space that gives us a measure of similarity between these concordance lines. How do we do that?

We proceed as we did before. We first exclude all the stop words: the pronouns, the articles, auxiliaries and prepositions. Then, in order to create a semantic representation of that very concordance line, we look up its context items in the type-based semantic vector space that we created earlier. For concordance line 1 , we look up the context items advantage, drugs, toxic and materials. All of these words are represented in the type-based semantic vector

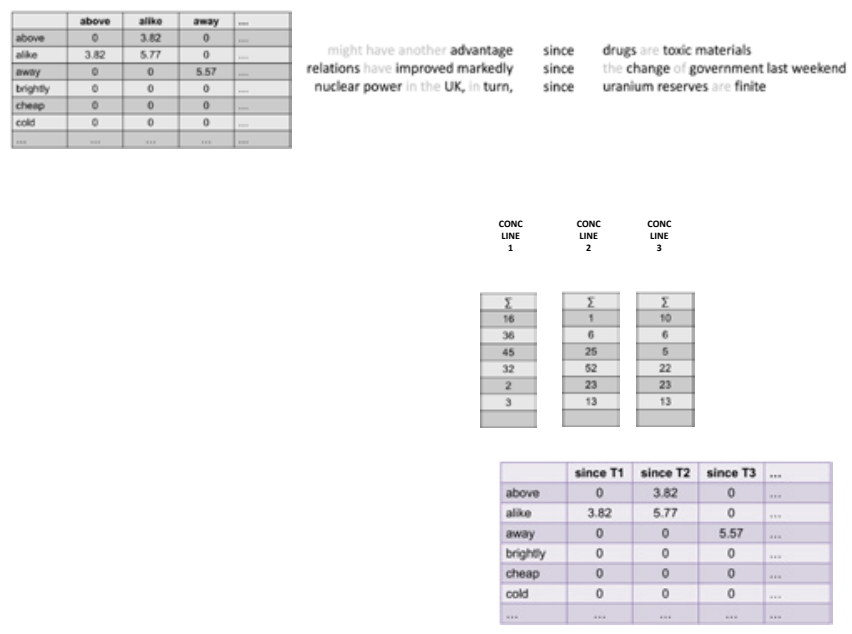

FIGURE 29 

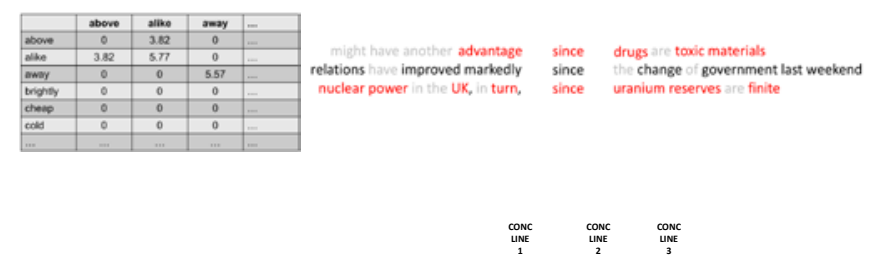

temporal since

vs.

causal since
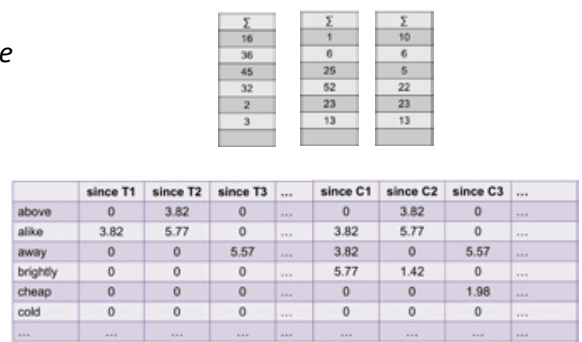

FIGURE 30

space. We just pull their context vectors out of the large vector space, and we create an average of their collocate vectors. That process yields a representation of our concordance line 1 in the analysis.

We then do the same for concordance line 2, for line 3 and for line 4, and so on and so forth, until we have a table that represents all lines of our concordances. With that kind of table, we can now create a visualization of a semantic space that is populated not by different word types, but rather by different concordance lines.

Let me tell you something about the three concordance lines you see here. They're all uses of since, but there is actually a semantic difference between the second example and the other two. The second since, "since the change of government last weekend" carries temporal meaning, and the first and the third express causal meaning. The semantic difference between temporal and causal meaning actually shows up in a visualization of a larger concordance of since.

In this graph here, you see little black dots and little red dots. The red dots are causal uses of since and the black dots are temporal uses of since. Each dot represents a concordance line. The positions of these dots in the graph have been calculated on the basis of their second order collocates, i.e. the context vectors of the words that are present in each concordance line. What you see in this graph is that there is some overlap. There is no perfect separation of temporal and causal since, but what you do see is that there is some structure in the graph. The red dots that represent causal since are more toward the right of the 


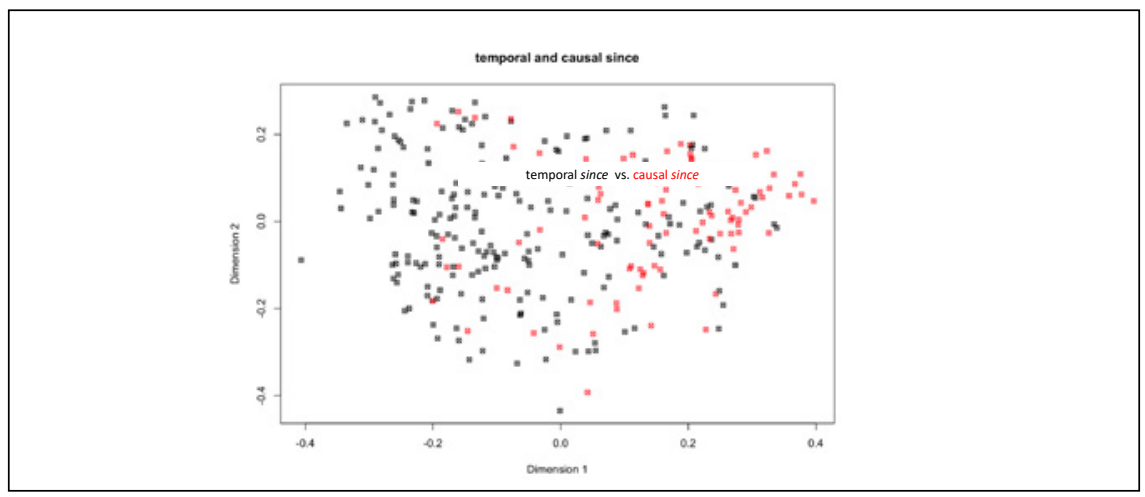

FIGURE 31

graph. On the left is a large area that is almost exclusively populated by black dots. In other words, with a token-based semantic vector space we can distinguish between two grammaticalized items, temporal since and casual since.

This is the point where I thank you for your patience, because this observation finally brings us back to asymmetric priming.

Data of this kind can be exploited to test the asymmetric priming hypothesis. Causal since is a case of secondary grammaticalization. Temporal since is the source that should, according to the asymmetric priming hypothesis, prime causal since, but not vice versa. According to the asymmetric priming hypothesis, if two instances of since follow one another, switches from temporal to causal should be more frequent, because time should make you think of causality but not the other way around. In every instance in which A turns into $B$, A should prime B, but not vice versa. The change from temporality to causality is exactly that kind of phenomenon.

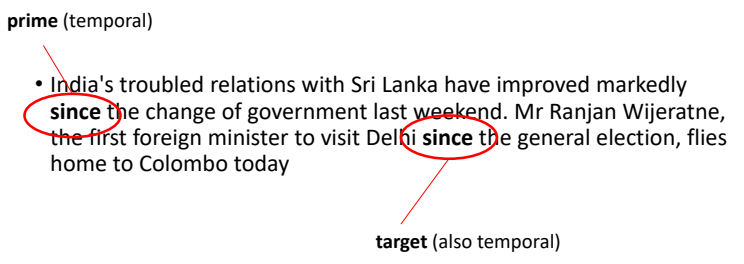

FIGURE 32 
The red and black dots that you saw in the graph earlier came from examples where two sinces were following each other. We took corpus examples such as the one shown on this slide: "India's troubled relations with Sri Lanka have improved markedly since the change of government last weekend". That sentence contains the first since. The following sentence contains another token of since: "Mr Ranjan Wijeratne, the first foreign minister to visit Delhi since the general election, flies home to Colombo today". The example thus involves two instances of since, and in this particular example, both are temporal. The first since is what we call the prime. That example expresses time. The second since is the target, and it also carries temporal meaning. Both sinces in this cases express the same type of meaning.

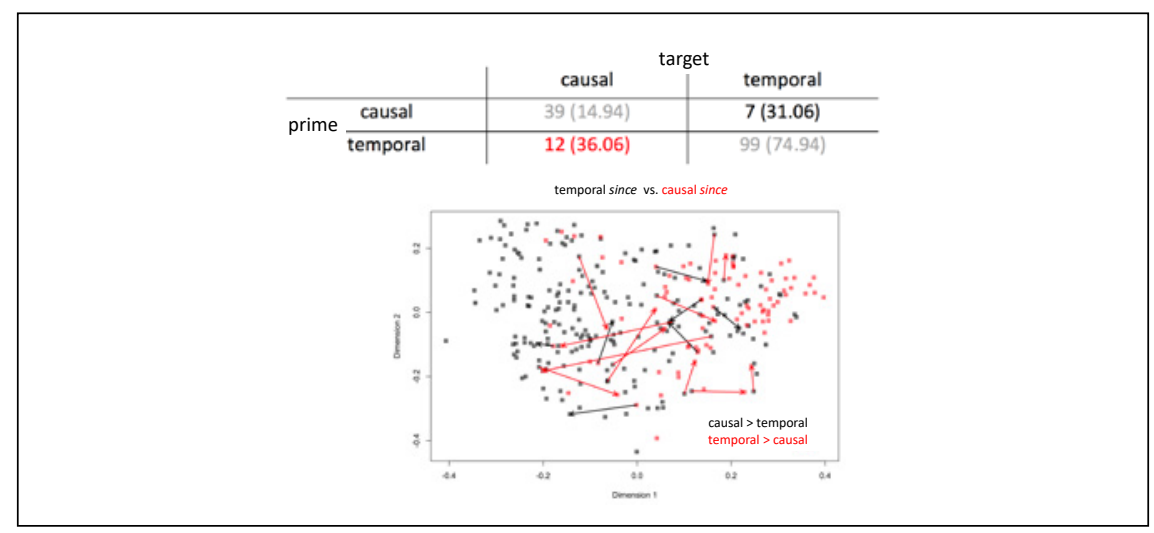

FIGURE 33

The question now is, when we have pairs like this, do we see switches from temporal to causal more often than we see switches in the other direction? This is something that you can easily count.

Let's first look at the table that you see right above the graph.

The numbers that you see are the observed frequencies. In brackets behind that, you see the expected frequencies. Let us examine the switches from temporal to causal. In our data, we observe 12 switches from temporal to causal, but we would have expected 36 , given the overall distribution of causal and temporal since. The asymmetric priming hypothesis predicts that switches from causal to temporal should be underrepresented, which is confirmed by our data. But the most important information in this table is that most pairings are sequences from causal to causal and from temporal to temporal. We observe 99 instances of two temporal instances of since, but we would have expected only 74 . We find 39 pairings that go from causal to causal, but we would only have expected 14 . 
A second piece of evidence follows from the graph with the token-based semantic vector spaces. In the graph, I have connected a number of pairs of data points with arrows. Red arrows indicate changes from temporal to causal, as predicted by the asymmetric priming hypothesis. Black arrows represent changes from causal to temporal. According to the asymmetric priming hypothesis, we would expect many more red arrows than black arrows. We would expect the arrows to pattern in a certain way, namely, we would expect them to start on the left side of the graph, which represents temporal meaning, to the right side of the graph, which represents causal meaning. That expectation is not met, as the arrows point towards different directions. This means that also our corpus-based analysis did not produce any evidence to support the asymmetric priming hypothesis.

You might be skeptical about the example of temporal and causal since, since it is just one example, and furthermore a case of secondary grammaticalization. In order to test that, we performed the same kind of analysis for other pairs of grammatical words and their lexical counterparts.

In this graph, we see a contrast of the lexical verb used and the grammaticalized habitual marker used to. The black data points represents lexical tokens, the red ones are habitual tokens. The analysis distinguishes nicely between them. The habitual data points show up at the left side of the graph and the lexical ones take up most of the graph. Again, when we look at the number of switches from lexical to habitual and from habitual to lexical, there is no significant difference between those. Neither the direction of the arrows nor the length of the arrows shows any difference, so there is no priming asymmetry towards habitual meaning.

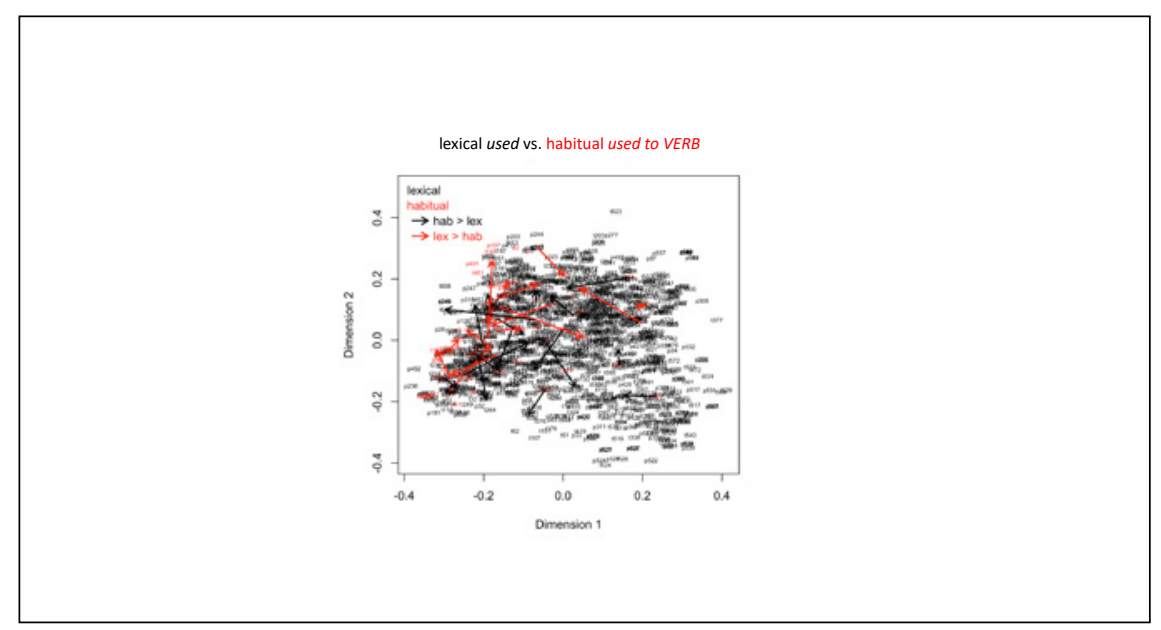

FIGURE 34 


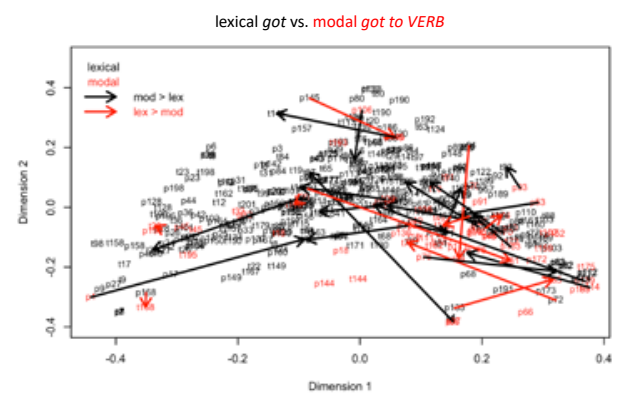

FIGURE 35

This slide shows another example. We performed the same analysis for lexical got versus modal got to and again the result is the same. There is no priming asymmetry towards modal got to.

In the last analysis, we compared deontic may as in "You may now kiss the bride" and epistemic may as in "That may be a mistake". The result is the same, no asymmetric priming effect.

What we do find in all cases though is that first, the semantic vector space allows us to discriminate rather neatly between the different meaning categories. Second, we find strong effects of within-category priming. Most pairings stay within their respective category, so that the sequences go from lexical to lexical and from grammatical to grammatical. This can be interpreted as a priming effect, but crucially not as asymmetric priming from lexical to grammatical.

With that I would like to come to my conclusions. The gist of the asymmetric priming hypothesis is that lexical going should prime grammatical be going to, whereas grammatical be going to should not prime lexical going. What actually happens is that lexical going strongly slows down grammatical be going to, but grammatical be going to does not slow down lexical going.

Why do we see this particular effect? The phenomenon of horror aequi suggests itself as an explanation. Processing the same form twice within the space of a few words is difficult, and speakers tend to avoid it. I have taken the liberty to construct a few sample sentences that we can subject to your intuition. The sentence "The boys need new shoes that we need to buy" involves two uses of need, the first is lexical and the second is grammatical. Here's another example, "You need to make a list of things you need". Again, two instances of need, but here the first is grammatical and the second is lexical. This strikes me as 


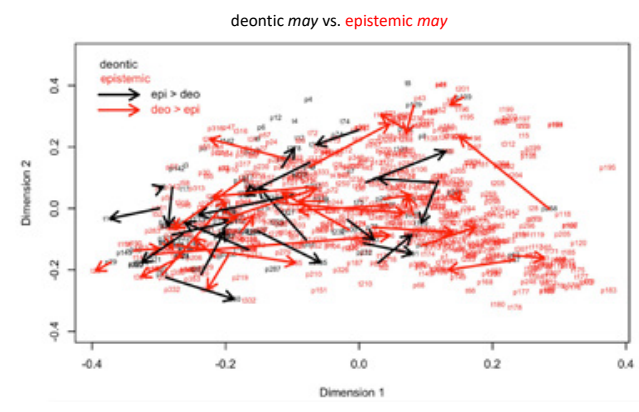

FIGURE 36

somehow better than the first one. If horror aequi has a role to play, then why should its effect be asymmetric? The best explanation that I can offer at this point is that semantic specificity may account for this.

When forms grammaticalize, their original lexical meaning fades. The more grammaticalized the form is, the less it will engender horror aequi effects. When I say "My parents have had a dog", which involves two instances of have, right next to one another, there is no horror aequi. Or consider "I am going to go there", in which two forms of go appear in close proximity, without any horror aequi effect. Compare that to "He used to use a typewriter", which is perhaps not terrible, but certainly not elegant, or "It happened to happen on a Tuesday", which sounds like someone deliberately played with language.

The first two are okay, because these are strongly grammaticalized and schematic constructions. The others are only weakly grammaticalized forms. Essentially, we believe that what we measured in the experiment was the degree of grammaticalization of our grammatical stimuli. For his dissertation, David Correia Saavedra has carried out a study of corpus-based measurements of grammaticalization. He has very interesting results that I hope you will find out more about soon.

Let me come to an end here. What we found in the corpus-based test of the asymmetric priming hypothesis is that lexical forms prime themselves, and grammatical forms prime themselves. We find self-priming of both lexical forms and grammatical forms, but no priming asymmetries towards the more grammatical variant.

In conclusion, both our experimental results and our corpus-based results detract from the asymmetric priming hypothesis. That is a little disappointing. 
I still think the hypothesis is a fascinating idea. Very importantly, this negative result does not call into question the power of priming as a force on language use. It also does not call into question the basic tenet of usage-based approaches that cognitive processes operating in the here and now shape language structure and language change. It just means that asymmetric priming is not the explanation for unidirectionality in semantic change. With that, thanks again for your attention. 